Sociologie et sociétés

\title{
Les processus de réforme et la structuration locale des systèmes. Le cas des réformes dans le domaine de la santé mentale au Québec \\ Reform Processes and the Local Structuration of Systems: The Case of Mental Health Reform in Quebec
}

\section{Deena WHITE}

Volume 25, numéro 1, printemps 1993

La gestion du social : ambiguïtés et paradoxes

URI : https://id.erudit.org/iderudit/001842ar

DOI : https://doi.org/10.7202/001842ar

Aller au sommaire du numéro

Éditeur(s)

Les Presses de l'Université de Montréal

ISSN

0038-030X (imprimé)

1492-1375 (numérique)

Découvrir la revue

Citer cet article

WHITE, D. (1993). Les processus de réforme et la structuration locale des systèmes. Le cas des réformes dans le domaine de la santé mentale au Québec. Sociologie et sociétés, 25(1), 77-97. https://doi.org/10.7202/001842ar
Résumé de l'article

L'article se veut un apport à la constitution d'une sociologie des processus de réforme. Nous ne prenons pas comme point de départ les programmes ou les acteurs de l'État mais, afin de rendre compte des effets diversifiés, pour ne pas dire arbitraires, des politiques sociales, nous nous intéressons plutôt à la façon dont les acteurs sociaux locaux s'approprient des processus de réforme. Nous présentons les résultats d'une étude de cas multiples axée sur les développements qui se sont produits dans le réseau des soins de santé mentale de trois localités du Québec, dans le contexte d'une réforme provinciale du système de santé mentale. Nous concluons en présentant un certain nombre d'hypothèses relatives à la structuration des sous-systèmes locaux face à l'intervention étatique. 


\title{
Les processus de réforme et la structuration locale des systèmes. Le cas des réformes dans le domaine de la santé mentale au Québec
}

\author{
DEENA WHITE \\ 'Traduit par Suzanne Mineau
}

\section{INTRODUCTION}

Cet article se veut avant tout un apport à une enquête spécifiquement sociologique sur les processus de réforme. Une telle approche diffère énormément de l'approche administrative, qui cherche à évaluer ou à expliquer l'efficacité relative des politiques et des programmes, ou de l'approche politique qui se préoccupe de la nature et de l'efficacité des organismes et des actions de l'État. L'intérêt de la sociologie pour la réforme se rattache avant tout au domaine plus vaste du changement social; nous nous interrogeons sur le rôle de l'État en tant qu'agent de changement social ainsi que sur la façon dont ce rôle recoupe et rejoint d'autres processus de changement social et avec quels effets. Dans ce contexte, nous ne pouvons prendre comme point de départ de notre enquête les programmes ou les acteurs de l'État, parce que cela laisserait supposer que les changements observés sont directement ou indirectement attribuables aux actions de l'État.

On considère généralement qu'une réforme relève de l'État ou des élites de l'État. On peut aussi faire une évaluation politique de la réforme et, dans ce cas, l'absence ou l'inconsistance des résultats peuvent être attribuées à des objectifs incohérents, à des barrières structurelles, à des résistances ou à des écarts dans le processus de mise en œuvre (voir par exemple Marks et Scott, 1991). Dans cet article, nous suivons une démarche inverse. Nous voulons adopter le point de vue que réclamait récemment Lisette Jalbert, soit «l'observation systématique des actions-réactions des agents de la base concernés par la réforme» (Jalbert, 1991, p. 254). Au lieu de considérer les actions entreprises par l'État québécois pour mettre en œuvre sa politique officielle de santé mentale adoptée en 1989, nous nous intéressons plutôt à la façon dont les acteurs locaux se sont appropriés les processus de réforme, parce que nous croyons que cette approche rend mieux compte des effets diversifiés, pour ne pas dire arbitraires, des politiques sociales dans les démocraties libérales contemporaines. 
Dans les démocraties libérales, la réforme constitue le mode de changement social le plus apparent ${ }^{1}$. Comme Boudreau (1987) l'a souligné, chaque génération donne le nom de réforme à ses propres politiques sociales. Pour nous, le concept de réforme signifie toute réorganisation sociale, politique ou économique impliquant l'intervention de l'État et se situant habituellement dans une sphère d'activité bien délimitée. Pourtant, il est trompeur d'appeler réforme une politique spécifique publiée et adoptée par un gouvernement, puisque cela équivaut à confondre réforme et discours. Il est vrai que le discours est un ingrédient important de la réforme, et, lorsqu'une politique officielle vient le légitimer, son influence peut être décisive. Cependant, plus qu'une politique, les processus de réforme englobent une série complexe d'activités visant à provoquer des changements dans la société. Un document politique peut représenter une de ces activités, et il vient s'insérer dans le processus au moment où le gouvernement est suffisamment assuré qu'un consensus accueillera son discours sur le sujet. Inévitablement, toutefois, les changements préconisés par la politique, c'est-à-dire les processus de réforme, ont commencé à apparaître bien avant cela.

Notre recherche est une étude de cas multiples axée sur les développements qui se sont produits dans le réseau des soins de santé mentale de trois localités du Québec. La toile de fond est la réforme provinciale du système de santé mentale qui débute, selon nous, en 1982, pour des raisons que nous expliquerons plus loin, et qui se poursuit encore aujourd'hui (en 1993). Bien que la politique gouvernementale dans ce domaine soit explicitée dans un document officiel qui n'a été publié qu'en janvier 1989 (Ministère, 1989), les grandes lignes de cette politique ont été arrêtées tout au long des années 80 , comme le démontrent, au niveau du discours, plusieurs Livres Blancs, nombre de documents administratifs moins officiels, les audiences de deux commissions parlementaires distinctes, plus de 150 mémoires présentés par les groupes professionnels et communautaires en cause ainsi qu'un nombre considérable d'analyses et de débats publics dans les revues et les journaux locaux. Toutefois, la période considérée est aussi une période d'action réformatrice, notamment de nouvelles stratégies de financement et de planification qui font intervenir les groupes communautaires dans un processus élargi de création de nouvelles ressources en santé mentale.

Notre étude vise à comprendre comment, pendant cette décennie, se sont réellement produits les nouveaux développements touchant l'organisation des soins de santé mentale dans des sites spécifiques ${ }^{2}$. Deux grandes tendances théoriques en analyse sociale ont influencé notre recherche: tout d'abord, la disparition des frontières rigides entre les analyses structurelles et les analyses de l'action sociale (Bourdieu, 1980; Giddens, 1981, 1984; Offe, 1985; Hindess, 1986, 1988); en second lieu, la transgression de l'opposition entre État et société civile (Held, 1989; Jalbert, 1992; Sales, 1991). Notre article veut saisir le processus de réforme à la base, décrire et interpréter les changements réels en

1. Par changement social, nous entendons des changements dans l'organisation discursive et pratique des systèmes sociaux. Bien que les changements sociaux ne prennent pas naissance dans les processus de réforme, ils sont généralement institutionnalisés par ces derniers. Parmi les auteurs qui ont inspiré cette façon de voir sans nécessairement la partager ou la défendre, mentionnons MARRIS (1987), chapitres 6 et 7; HINDESS (1986), p. 125; GIDDENS (notamment 1981). Pour ce qui est de certaines opinions sur le rôle de 1'État dans le changement social, mentionnons EVANS, RUESCHEMEYER et SKOCPOL (1985), TOURRAINE (1973) et URRY (1982).

2. Notre travail de recherche (janvier 1990 à mai 1993) s'est concentré sur cinq localités différentes, bien que nous n'en mentionnions que trois, parce que nous n'avions pas complété la collecte et l'analyse des données des deux autres au moment de soumettre cet article (juin 1992). Notre étude comparative a exigé des entrevues en profondeur avec huit à quinze acteurs clés dans chaque localité, ainsi qu'avec des administrateurs régionaux et provinciaux. Il a fallu aussi analyser les procès-verbaux des réunions de nombreux comités et groupes sociaux. L'auteure désire profiter de l'occasion pour mentionner la précieuse collaboration de Céline Mercier, co-chercheure. Elle désire aussi remercier, pour leur importante contribution, Francine Desbiens et Marie-Claude Roberge, ainsi que d'autres membres du Groupe de recherche sur les aspects sociaux de la prévention de l'Université de Montréal. Cette recherche était généreusement financée par le Programme national de recherche et de développement en santé de Santé Bien-Être Canada. On peut obtenir le protocole détaillé de cette enquête (WHITE, MERCIER ET DESBIENS, 1991). 
tenant compte de toute la gamme des agents sociaux en cause ainsi que des conditions structurelles de leur action. Sans négliger l'État, nous ne lui accordons pas a priori le premier rôle dans ce processus.

Nous commencerons pas exposer le cadre théorique qui nous a amené à nous intéresser aux dimensions locales des processus de réforme. Puis, après avoir esquissé les conditions globales de la réforme de la santé mentale dans les années 80 au Québec, nous relèverons les éléments importants des processus de réforme dans trois localités différentes. En comparant ces cas, nous pourrons ensuite avancer un certain nombre d'hypothèses liées à l'analyse sociologique de la réforme en tant que processus de changement social et aussi liées aux limites du rôle de l'État en tant qu'agent de changement social par rapport à d'autres acteurs sociaux.

\section{IMPORTANCE DU LOCAL DANS L'ANALYSE DES PROCESSUS ET DES RÉSULTATS D'UNE RÉFORME}

Les théories sociologiques sur l'État nous enseignent qu'il faut regarder au-delà ou "sous» l'État pour comprendre ses actions et son influence. D'un point de vue tant pluraliste que marxiste ou néo-marxiste, les politiques gouvernementales résultent des revendications de groupes sociaux puissants (voir par exemple Alford, 1975) ou des luttes de classe et de l'hégémonie incarnée par l'État (voir par exemple Gough, 1979; Offe, 1984). De plus, selon des théories récentes, les réformes mises de l'avant par l'État produisent dans la société des changements et des réactions sociopolitiques qui sont voulus aussi bien qu'imprévus; les réformes peuvent aussi influer sur la formation des groupes sociaux ainsi que sur le potentiel politique, sur les idées et sur les revendications de divers secteurs sociaux (Skocpol, 1985; Rueschemeyer et Evans, 1985; Hayek, 1973; Lowi, 1979). L'ensemble de ces opinions laisse croire que les frontières entre l'État et la société civile sont extrêmement poreuses (Held, 1989; Jalbert, 1992). Pour comprendre les processus de réforme, il nous faut considérer les multiples «actions et rétroactions ... de l'État vers la société et de la société vers l'État» (Jalbert, 1992, p. 84).

Franchissons maintenant un pas de plus en disant que l'opposition entre État et société civile, ou entre les concepts équivalents de centre et de local, n'est peut-être plus utile et peut même nous empêcher d'analyser en profondeur les processus de réforme dans les sociétés contemporaines. L'analogie que nous établissons entre les dualités centre/ local et État/société civile laisse voir que le mot «local " n'a pas ici le sens de «périphérique», mais qu'il désigne plutôt des conditions immédiates et proches englobant l'ensemble des conditions opérationnelles, même celles associées au «centre». Habermas soutient que l'interpénétration de l'État et de la société civile a progressé au point où leur synthèse constitue un niveau d'action sociale - le système - qui se surimpose au "vécu» privé de la population (Sales, 1991). Le système est alors constitué de la gamme complète des agents régulateurs interreliés des sphères organisationnelle et discursive qui se situent au niveau local des activités quotidiennes.

En outre, il est devenu très difficile d'établir une distinction entre les organisations qui représentent l'État ou agissent en son nom et les organisations qui se veulent autonomes et autorégulées, parce qu'il se produit une interpénétration de l'État et de la société civile, du centre et du local, même à l'intérieur de ces organisations (White, 1988). Les exemples ne manquent pas; ainsi, des établissements publics sous la gouverne de l'État, comme les hôpitaux, englobent des conseils d'administration élus et des groupes professionnels qui peuvent s'opposer férocement à l'intervention de l'État et défendre de vastes zones de prises de décision autonomes. De même, des organismes indépendants et sans but lucratif sont financés et régis par l'État à des degrés divers, et en vertu de certaines politiques contemporaines de décentralisation et de «partenariat», ils peuvent collaborer à des politiques parrainées par l'État et à des opérations de planification générale.

Dans un tel contexte, les oppositions «État versus société civile» et «centre versus local» peuvent être trompeuses lorsqu'elles sont utilisées dans des recherches empiriques 
sur les processus de changement social. Tout d'abord, cette dualité analytique peut masquer la complexité réelle des acteurs sociaux en nous forçant à les placer dans une sphère ou dans l'autre, et à leur accoler automatiquement des caractéristiques, comme «intérêts » ou «pouvoir», associées à cette sphère (Hindess, 1982). En deuxième lieu, on présume trop facilement qu'il existe une relation hiérarchique entrè les deux sphères opposées, si bien par exemple qu'une transformation observée localement (dans un organisme ou dans une localité donnée) tend à être expliquée par des événements qui se produisent dans des lieux plus au centre ou plus globalisants comme l'État ou la grande entreprise. Pourtant, tout lieu a une existence distincte qui ne peut être prise en compte qu'en considérant toute la gamme unique (et parfois contradictoire) de ses discours, ressources, acteurs et rapports (Hindess, 1986). Par conséquent, aucun lieu d'action sociale ne peut être expliqué par référence à un autre lieu. Contrairement à ce que laisse souvent croire l'utilisation de concepts dichotomiques comme État/société civile ou centre/local, chaque élément de la paire ne constitue pas simplement un aspect d'un même processus.

En troisième lieu, de telles dualités analytiques peuvent être trompeuses parce que les conditions et les rapports locaux, ou immédiats, ne sont jamais exclusivement locaux, puisque des acteurs du «centre», par exemple les agents étatiques, sont inévitablement ancrés dans des espaces locaux et soumis aux conditions locales. D'autre part, des changements dans les formes d'action sociale ont nécessairement leur point de départ dans les conditions proches que connaissent les acteurs sociaux et dans les moyens dont ils disposent pour interpréter ou expliquer ces conditions (Foucault, 1980; Giddens, 1987) 3 . Toutefois, les discours, les ressources et même les acteurs spécifiques que l'on retrouve dans un certain lieu traversent en fait un éventail de lieux très vaste et diversifié et hypothétiquement illimité incluant des sites locaux supposément centraux comme des services gouvernementaux. Il semblerait donc que la seule chose qui puisse expliquer les réformes, même les plus fondamentales, soit les conditions et les acteurs qui caractérisent des espaces locaux d'action sociale ainsi que la nature des liens de médiation entre ces lieux (Bourdieu, 1980; Hindess, 1986).

Soulignons une fois de plus que le local ne représente pas un système d'action autonome. Ainsi, il ne fait aucun doute que les stratégies de l'État contemporain en matière de régionalisation, de décentralisation, de participation, de concertation et de partenariat inventent en fait des espaces locaux (Jalbert, 1991, 1992; Godbout, 1983; Valarié, 1988, Klein, 1989). Par contre, l'État n'est pas en mesure par la suite de maîtriser le fonctionnement de ces lieux. D'autre part, l'accent que nous mettons sur la dimension locale des processus de réforme ne doit pas être interprété comme un cri de ralliement en faveur du «culte du local» (Mowbray, 1985; Tomlinson, 1991), puisque nous ne portons aucun jugement normatif. Nous ne prétendons pas que les réformes locales sont plus désirables ou moins désirables, mais plutôt que les événements qui se produisent au sein de ces lieux ainsi que les rapports entre eux sont des clés qui permettent de comprendre le changement social.

Notre perception du local réfère donc aux conditions spécifiques, discursives et non discursives, dans lesquelles se situent les décisions et les actions liées à un domaine donné. Cela implique qu'une réforme, comme n'importe quel processus de changement social, n'est pas une opération globale et unitaire, mais une opération fragmentée. Les soi-disant «centres» (par exemple, les ministères de l'État ou les autorités régionales) sont eux-mêmes des sites locaux de réforme, et les stratégies adoptées à leur niveau ne suffisent aucunement à expliquer les résultats sur le terrain. Par conséquent, pour

3. HINDESS $(1986,1988)$ corrobore ce point de vue lorsqu'il analyse les « intérêts objectifs » à titre de catalyseurs de l'action sociale et qu'il conclut à l'impossibilité de déduire les intérêts d'un acteur à partir de sa position sociale: «il n'est pas possible que des intérêts (ou des normes ou des valeurs) fonctionnent comme de simples courroies de transmission entre la structure sociale et les décisions des acteurs. Les intérêts n'ont des conséquences que dans la mesure où ils interviennent dans les délibérations des acteurs et qu'ils contribuent à leur fournir des motifs d'action » $(1988$, p. 110). 
expliquer les effets des processus de réforme, il est nécessaire de repérer: (1) les conditions communes à toute une série de sites locaux; (2) la configuration des acteurs locaux ainsi que les enjeux immédiats suscités par leurs interactions; (3) la nature des liens de médiation entre un site et d'autres agissant dans le même domaine ou dans des domaines connexes; enfin, (4) les stratégies grâce auxquelles les acteurs locaux s'approprient le processus de réforme, c'est-à-dire les moyens par lesquels ils prennent des décisions et agissent dans le domaine de la réforme.

Revenons maintenant à la question de la localité en tant qu'elle constitue, d'une part, un lieu délimité de réforme et, d'autre part, un site se prêtant à l'analyse d'une réforme sociale. En règle générale, les localités ${ }^{4}$ sont artificielles, au sens qu'elles résultent de stratégies administratives, comme la régionalisation et la décentralisation, qui délimitent et agencent les territoires géographiques dans le but de répartir, de coordonner et de surveiller les «ressources» humaines et matérielles. En fait, c'est précisément parce qu'elles ont été désignées au niveau administratif, comme site de mise en œuvre d'une politique, que les localités peuvent constituer des lieux convenant à l'analyse des processus de réforme, puisqu'on peut s'attendre à ce qu'elles soient soumises à une série de conditions relativement cohérentes, du moins en ce qui concerne le domaine de la réforme en question. Toutefois, les acteurs locaux ne se constituent pas nécessairement eux-mêmes en système d'action sociale à l'intérieur des frontières du territoire désigné. Dans deux des cas que nous examinerons plus loin, on a changé au moins une fois les frontières des localilés étudiées pendant la période couverte par notre recherche, au terme de négociations, entre les acteurs locaux et l'organisme gouvernemental concerné, relativement aux caractéristiques géographiques et communautaires du territoire.

Cela nous amène à un dernier point lié à l'importance du local dans l'analyse des processus de réforme, soit la distinction entre la localité et la communauté, deux réalités qui coïncident rarement dans les sociétés occidentales contemporaines. Dans la littérature sociologique, le mot "communauté " a donné naissance à des douzaines de définitions, dont certaines qui sont axées sur la référence au système et au territoire, définitions qui semblent correspondre au concept de localité dont nous traitons ici (Médard, 1961). Cependant, nous avons opté pour une nette différenciation des deux notions. Par communauté, nous entendons uniquement les frontières subjectivement significatives de l'inclusion et de l'exclusion par rapport à un groupe (Weber, 1978; Cohen, 1985). Le lien entre la communauté et la localité, lorsqu'il existe, est en grande partie arbitraire et symbolique. Cependant, les mots "communauté " et "communautaire» occupent beaucoup de place dans le contexte empirique de notre recherche sur les processus de réforme de la santé mentale au Québec; ils réfèrent à un ensemble spécifique d'approches ou de méthodes de planification et de distribution de services dans le domaine de la santé mentale ${ }^{5}$. Nous analyserons la teneur de ces discours «communautaires» dans la section suivante consacrée aux conditions globales de la réforme.

\section{ILA RÉFORME DES SERVICES DE SANTÉ MENTALE AU QUÉBEC}

Le contexte local des processus de réforme englobe certaines conditions qui couvrent plusieurs lieux. Dans le cas des réformes de la santé mentale, on trouve dans les sociétés européennes et américaines certaines de ces conditions globales, comme les mouvements prônés par l'État en faveur de la désinstitutionnalisation des aliénés et la mise sur pied de stratégies «communautaires» à cette fin (Scull, 1984). Toutefois, la nature de ces développements mondiaux et le moment où ils se produisent diffèrent d'une société à l'autre; ils atteignent et influencent les espaces locaux spécifiques de la réforme

4. Le mot localité réfère généralement à un espace territorial. D'autres espaces locaux (comme par exemple les organismes, les bâtiments, les corps humains) peuvent aussi constituer des lieux d'action sociale.

5. Pour une analyse des innombrables usages du mot «communautaire» dans le domaine de la santé mentale et de leurs liens (parfois lointains) avec les concepts standards de la «communauté » en sociologie, voir WHITE (à paraître). 
de façon fragmentée. Par conséquent, on peut mieux saisir la nature de ces influences globales sur les localités québécoises en les présentant dans le contexte du Québec; celui-ci s'est doté d'un système de gestion et de communication relativement unifié qui lui permet de filtrer les tendances globales dans le domaine de la santé mentale, et ce système est commun aux trois cas de réforme que nous analyserons par la suite.

\section{CONDITIONS GLOBALES QUI ONT PRÉCIPTTÉ LA RÉFORME EN SANTÉ MENTALE AU QUÉBEC ${ }^{6}$}

Les conditions qui ont donné lieu à la réforme de la santé mentale au Québec sont triples: l'état du système des services de santé avant le début des processus de réforme; les conditions sociales, économiques, politiques et culturelles qui ont accéléré certains types de changements dans la société tout entière, et des conditions liées plus particulièrement au domaine de la santé mentale.

Disons d'abord que toute analyse du système général de santé du Québec doit partir des réformes Castonguay officiellement adoptées en 1971. Cette série de lois a remplacé un ensemble chaotique d'institutions «communautaires» (appartenant en grande partie à des communautés religieuses) par un réseau complet d'établissements publics englobant tous les hôpitaux généraux et spécialisés, la plupart des centres d'accueil ainsi que tous les centres de services sociaux. Elle a aussi créé plusieurs structures nouvelles comme les CLSC (Centres locaux de services communautaires) et les conseils régionaux. Avec l'assurance-maladie universelle et l'assurance-hospitalisation universelle (cette dernière déjà en vigueur depuis une décennie), le nouveau réseau visait à répondre à tous les besoins de la population en matière de soins de santé et de services sociaux. Par contre, il ne considérait pas les troubles mentaux comme une question à part, mais comme une maladie au même titre que les autres.

En vertu de ce système, la plupart des nouveaux patients psychiatriques étaient admis dans les hôpitaux généraux assurant des soins de courte durée, ostensiblement dans le but de favoriser la non-institutionnalisation des personnes souffrant de maladie mentale et de permettre la désinstitutionnalisation des patients recevant des soins de longue durée dans les hôpitaux psychiatriques. En dépit de la mise sur pied de cliniques externes dans de nombreuses localités et de l'augmentation, sous la surveillance des centres de services sociaux, de la création de familles d'accueil spécialisées, la désinstitutionnalisation des hôpitaux psychiatriques a été lente. Dans les faits, le système a médicalisé les services de santé mentale, tandis que les services psychologiques et sociaux destinés aux personnes non hospitalisées ou désinstitutionnalisées sont demeurés rares, sinon inexistants, dans les secteurs public et privé.

De plus, les services de consultation externes et les familles d'accueil n'étaient pas en mesure de faire face aux difficultés d'intégration sociale des patients psychiatriques. Cela nous amène à notre deuxième point: de plus en plus de personnes souffrant de troubles mentaux graves et chroniques ont commencé à être soignées à titre de patients externes et uniquement sur le plan médical à l'aide de tranquillisants et de neuroleptiques; on a donc vu apparaitre peu à peu des problèmes psychosociaux qui touchaient non seulement ces personnes, mais un éventail élargi de groupes sociaux: augmentation de l'errance et de la délinquance, toxicomanie et alcoolisme, rupture des familles, recours massif aux services d'urgence et syndrome de la porte tournante, selon lequel les crises psychosociales ou psychotiques entrainent des réhospitalisations répétées. Dans les années 70 et au début des années 80, ces phénomènes sont devenus de plus en plus courants, et tous les professionnels de la santé mentale ainsi que les médias et les groupes de pression se sont mis à réclamer une réforme. L'aile la plus radicale de ce mouvement au Québec était le Regroupement des ressources alternatives en santé mentale, établi en

6. Pour une analyse complète de ces conditions et des mouvements connexes au Québec, voir WHITE et MERCIER (1991a). 
1982 en tant qu'association de groupes d'entraide, de groupes de défense des droits et d'organismes à but non lucratif offrant des programmes de soutien et de thérapie dans une perspective anti-psychiatrique ${ }^{7}$.

La réforme Castonguay dans son ensemble, et plus particulièrement les politiques de désinstitutionnalisation et de non-institutionnalisation, faisaient partie des stratégies de l'État pour créer un système rationnel de santé au Québec tout en limitant les coûts, mais ce second impératif a pris une place déterminante pendant la récession du début des années 80. En 1982, le gouvernement a coupé dans les budgets des services de santé et des services sociaux. Les pressions se sont accrues pour que diminue le nombre de lits dans les coîteux établissements de soins de longue durée, surtout les hôpitaux psychiatriques. En 1984, le gouvernement a même placé sous tutelle le plus important hôpital psychiatrique de la province parce qu'il ne désinstitutionnalisait pas ses patients à un rythme assez rapide. À la même époque, on notait, au sein du gouvernement et des groupes professionnels, un début d'intérêt pour le développement de nouvelles formes de services de santé mentale, en dehors du réseau public et syndicalisé qui avait été créé à peine dix ans auparavant. Cet intérêt pour un désengagement de l'État et pour des solutions «communautaires » n'a fait que croître avec l'élection d'un gouvernement néo-conservateur en 1986.

Finalement, dans le domaine de la santé mentale, il s'est produit au Québec, en 1982, quelques événements précis qui peuvent être considérés comme des catalyseurs prochains de l'amorce d'une réforme systématique. Beaucoup de ces événements sont directement ou indirectement liés à la nomination d'une nouvelle directrice intérimaire des services de la santé mentale au ministère de la Santé. Ni psychiatre ni même médecin, cette clirectrice était une jeune administratrice personnellement engagée dans le mouvement «communautaire». Sa nomination a donné à de nombreux individus et groupes l'occasion de promouvoir des changements avec l'appui tactique du Ministère. Par exemple, la directrice intérimaire a réussi, en dépit des mesures d'austérité financière, à obtenir des fonds pour la mise sur pied d'un certain nombre de ressources communautaires en santé mentale, notamment le Regroupement des ressources alternatives, et elle a soutenu quelques colloques provinciaux ainsi que d'autres événements importants axés sur les ressources alternatives en santé mentale. En raison de ces circonstances spéciales qui créaient une «structure politique opportune ${ }^{8}$ » pour divers groupes contestataires et certains réformateurs, en raison aussi de la conjoncture économique, politique et sociale que nous avons décrite précédemment, nous sommes amené à faire de 1982 l'année où a débuté au Québec le processus de réforme de la santé mentale.

\section{LE DISCOURS «COMMUNAUTAIRE» ET LES STRATÉGIES SOCIALES}

Nous mettons le mot «communautaire» entre guillemets parce qu'il s'agit d'un concept dont la signification est à la fois essentielle et trompeuse dans le contexte de notre étude. Tous les intéressés s'entendent pour dire que la réforme favorisait un système axé

7. L'anti-psychiatrie est le nom donné au mouvement international qui est apparu dans les années 60 et dont les vues sont associées à certains auteurs comme GOFFMAN (1961), SZASZ (1977), FOUCAULT (1961, 1963) et COOPER (1967). Le mouvement condamne, à titre de méthode de contrôle social, la médicalisation des problèmes psychosociaux, par exemple, les méthodes de diagnostic psychiatrique, l'attention accordée uniquement aux symptômes et le traitement par la médication. Ses partisans partagent deux grandes valeurs : tout d'abord, importance de l'aspect psychosocial par opposition à l'étiologie individuelle et bio-médicale pour ce qui est de la nature des problèmes «psychiatriques»; en second lieu, prise en charge et contrôle des moyens de soutien par ceux qui ont des problemes psychosociaux.

8. Cette expression utilisée par S. Tarrow réfère a «l'ensemble des possibilités offertes aux contestataires par le système politique, et, par conséquent, par des facteurs comme son degré d'ouverture, sa souplesse et la nature de ses élites » (MELUCCI, 1989, p. 22). Dans le cas présent, le caractère opportun provient. de la structure disjointe du ministère de la Santé et des Services sociaux ainsi que des tendances radicales de nombreux professionnels des échelons inférieurs, notamment, par exemple, de la directrice intérimaire des services en santé mentale. 
sur la communauté et que cette orientation sous-entendait une distanciation par rapport à des soins essentiellement médicaux et axés sur l'hôpital. Par contre, nous percevons au moins trois discours communautaires différents utilisés sans cohésion par les différents acteurs sociaux (White, à paraitre):

(1) un discours politico-administratif qui assimile communauté et société civile et dans lequel les services communautaires sont ceux offerts en dehors du réseau d'établissements régi par l'État;

(2) un discours de géographie humaine ou «écologique»qui assimile communauté et localité et dans lequel les services communautaires sont ceux qui sont très proches des usagers et qui se révèlent, par conséquent, ouverts aux besoins locaux et d'accès facile pour la population locale;

(3) un discours sociologique qui assimile communauté et solidarité et dans lequel les services communautaires sont ceux qui répondent à une tradition d'entraide et de respect mutuel et évitent de façon explicite le contrôle professionnel par le biais de l'institutionnalisation, de la «psychiatrisation » ou de la médicalisation des troubles psychosociaux.

De plus, il n'est pas possible d'attribuer un de ces trois discours à un acteur social en particulier. Ainsi, le Regroupement des ressources alternatives du Québec a recours à un discours anti-psychiatrique qui repose sur une analyse sociologique de la réglementation de la santé mentale. Par contre, il utilise avec autant de verve un langage politicoadministratif pour réclamer l'autonomie administrative et fonctionnelle des organismes communautaires par rapport à l'État. Enfin, certaines factions du mouvement demeurent fortement attachées à la planification et à la prise de décision locale. Le discours communautaire le plus élémentaire est peut-être celui qu'utilisent bien des professionnels de la santé mentale qui œuvrent dans le domaine médical, comme certains travailleurs sociaux et certains psychiatres. Ils tendent à considérer toute ressource non institutionnelle comme une ressource communautaire, dans certains cas, même l'hôpital local.

La politique gouvernementale emprunte à tout l'éventail des discours communautaires, sans pourtant utiliser ces discours de la même façon que la plupart des groupes communautaires. Plutôt que les professionnels, elle tend à valoriser de plus en plus les intervenants «naturels», comme les groupes d'entraide, les membres de la famille et les bénévoles de la communauté, conformément à la tradition voulant que la communauté constitue un groupe solidaire. Certains efforts sont également faits pour promouvoir la création d'organismes de services indépendants et sans but lucratif qui remplaceraient des services publics trop coûteux. F. Boudreau a soutenu que les stratégies communautaires présentées dans les documents gouvernementaux des années 80 constituaient "un mélange unique d'individualisme et de collectivisme» (Boudreau, 1987, p.38). Toutefois, on peut aussi les considérer comme l'incarnation des valeurs néo-conservatrices qui dominent dans bien des gouvernements occidentaux actuels et qui allient la croyance en la responsabilité personnelle à la confiance en l'entraide et en la solidarité communautaire (Thorpe, 1985).

Bien qu'ils puissent peut-être acquérir un sens conservateur entre les mains des stratèges des États contemporains, le vocabulaire et les arguments en faveur de la réforme de la santé mentale au Québec sont apparus à l'extérieur de l'État, lors des remises en question du système public et psychiatrique par des groupes professionnels et par des mouvements sociaux. Toutefois, les répercussions de ces remises en question sont imputables en grande partie à une structure politique opportune créée par un gouvernement en difficulté financière ainsi qu'aux événements fortuits qui se sont déroulés dans les services administratifs en cause. Entre 1982 et 1989 (année de l'adoption de la politique officielle en santé mentale), on a donc vu apparaître un nombre significatif de petits programmes novateurs, entre autres dans le domaine de l'hébergement, programmes destinés à des personnes désinstitutionnalisées ou non institutionnalisées chez qui on avait diagnostiqué des troubles mentaux graves et chroniques, et qui étaient parrainées par des professionnels institutionnels, par des organisateurs communautaires ou par des réformateurs militants dans diverses localités. L'absence de cohésion dans le discours 
communautaire en faveur d'une réforme de la santé mentale au Québec explique en partie la possibilité de résultats et de processus différents dans différentes localités.

\section{LA RÉGULATION DU PROCESSUS DE RÉFORME}

La politique officielle publiée par le gouvernement du Québec en 1989, dans la foulée d'un Livre Blanc et d'audiences parlementaires, avait trois grandes orientations. Premièrement, elle exigeait la poursuite rapide de la désinstitutionnalisation systématique, mais avec un meilleur suivi des ex-patients grâce à des «plans de services individualisés» obligatoires. Deuxièmement, elle faisait des ressources communautaires les principales sources d'aide dans la communauté et réservait les traitements de courte durée aux hôpitaux et aux services spécialisés. Troisièmement, elle décentralisait l'autorité et le contrôle budgétaire en matière de services de santé mentale pour les confier à des conseils régionaux; ceux-ci disposaient d'une année pour soumettre un plan régional d'organisation des services destiné à assurer un éventail complet de services dans chaque région. Le premier objectif de la politique ne nous intéresse pas directement puisqu'aucune des localités que nous avons étudiées n'a participé à la désinstitutionnalisation, faute d'abriter un établissement psychiatrique sur son territoire ou à proximité. Pour ce qui est de la promotion des solutions communautaires pour les problèmes de santé mentale, le programme officiel mis de l'avant dans la politique était le suivant:

Il importe de reconnaitre que la contribution des services publics représente, dans la réponse aux besoins des personnes, un complément à celle des personnes elles-mêmes, de leur famille, de leurs proches et de la communauté. L'apport des organismes issus de la communauté est particulièrement manifeste en santé mentale. Pour favoriser le maintien dans le milieu de vie et la réintégration sociale, il devient essentiel d'appuyer ces groupes et d'accueillir les solutions qu'ils proposent. Le Ministère veut ainsi reconnaitre le potentiel des organismes communautaires, préciser le soutien qui doit leur être accordé pour remplir les rôles qu'ils se sont donnés, et confirmer leur participation, dans chaque région, à la conception et la mise en ouvre des services (Ministère, 1989, p.49).

Pendant la première année de mise en ouvre (1990-91), deux types de programmes axés sur la communauté ont bénéficié d'un faible financement nouveau: les groupes d'entraide et la relève des familles. Pendant la seconde année (1991-92), l'État a financé dans chaque région un programme de promotion de la santé mentale et de prévention, mais, en règle générale, ces programmes ont été organisés par les services régionaux de santé communautaire ou menés en étroite collaboration avec eux ; bien qu'étant multidisciplinaires du point de vue de leur approche professionnelle et de leur personnel, ces services étaient néanmoins rattachés à un hôpital. Aucun financement nouveau n'a été alloué à la création d'organismes communautaires de services capables de concurrencer les services psychiatriques fournis par les divers professionnels œuvrant dans le réseau public de la santé et des services sociaux. En outre, comme tous les fonds alloués par le Ministère aux services communautaires de santé mentale sont maintenant consolidés au sein d'un seul bureau, les gestionnaires trouvent, grâce à d'habiles manœuvres innovatrices, moins d'argent pour les groupes communautaires qu'au cours de la décennie précédant la réforme, lorsque les manœuvres créatrices étaient toujours possibles. Le gouvernement semble s'attendre à ce que les conseils régionaux financent les nouveaux services par la réallocation des fonds alloués aux hôpitaux psychiatriques.

De tous les aspects de la politique officielle, c'est sans doute la décentralisation qui a eu le plus d'impact sur les processus de réforme locaux. Chaque Conseil régional était tenu d'établir un plan quinquennal décrivant de quelle façon il mettrait une gamme complète de services en santé mentale à la disposition de sa population. Le Conseil devra également gérer le budget accordé par le Ministère pour la mise en œuvre de ce plan. Le 
premier plan devait être élaboré en collaboration avec un comité régional tripartite regroupant en nombre égal des représentants (1) des établissements régionaux publics fournissant des services en santé mentale, (2) des organismes communautaires œuvrant dans ce domaine et (3) des «différentes communautés de la région et des organisations engagées dans des secteurs dont les actions peuvent avoir une influence sur la promotion de la santé mentale, la prévention et la réinsertion» (Ministère, 1989, p. 54), par exemple, les commissions scolaires, les policiers, les municipalités et, à l'occasion, des groupes communautaires œuvrant dans des domaines comme la toxicomanie et la violence conjugale. En plus, plusieurs conseils régionaux se sont jusqu'à un certain point décentralisés ou régionalisés à l'intérieur de leur propre territoire afin de favoriser la participation, l'apport ou la consultation des différentes localités.

Les comités tripartites ont constitué d'importants lieux de conflits et de stratégies dans les processus de réforme, et ils ont évidemment fonctionné de façon différente dans chaque région; tout dépendait du mode de fonctionnement du Conseil régional, de la position des acteurs régionaux, des rapports entre ces acteurs et des stratégies adoptées. Néanmoins, nous démontrerons dans les sections suivantes que, même dans les régions où différentes localités ont été soumises au même processus de planification tripartite et où il y a eu très peu de décentralisation interne, les effets du processus ont été très différents. En fait, nous pensons que la politique provinciale et le processus de planification régionale se sont superposés aux processus de réforme locaux qui existaient depuis un certain temps et qui ont déterminé les résultats de la politique.

\section{BANLIEUE OUEST}

La banlieue ouest est un vaste territoire voisin de Montréal et sa population, qui appartient majoritairement à la classe moyenne, affiche un des taux de croissance les plus élevés de la province. Les services de santé et les services sociaux publics n'ont pas augmenté au même rythme. Il y a un seul petit hôpital général qui éprouve des difficultés à recruter du personnel spécialisé, en partie parce qu'il ne maintient aucun lien avec les universités de la ville de Montréal. L'hôpital possède une unité psychiatrique, où travaille à temps partiel un petit nombre de psychiatres traditionnels et qui a été peu touchée par le discours pro-communautaire. Elle est desservie par l'un des centres de services sociaux de l'île de Montréal. Il existe aussi deux CLSC, mais aucun n'offre un programme de services en santé mentale, même si l'un d'eux a contribué à mettre sur pied le premier organisme communautaire en santé mentale du district à la fin des années 70 , soit un «club» administré par des bénévoles. Les ressources en santé mentale par rapport à la taille de sa population font de cette banlieue la sous-région la plus démunie de l'île de Montréal.

Les organismes bénévoles locaux sont traditionnels et typiques de la classe moyenne: groupements de bienfaisance, groupes de femmes associés aux Églises et aux autres institutions locales. Paradoxalement, à côté de cette tradition communautaire consensuelle, on trouve des familles qui luttent au nom de leurs membres ayant des problèmes psychiatriques en utilisant un discours militant et revendicateur. Ainsi, les leaders locaux, d'une part, mettaient sur pied des programmes traditionnels de bénévolat, comme des visites périodiques aux patients psychiatriques vivant dans la communauté, et, d'autre part, organisaient des campagnes de lettres adressées aux journaux, aux députés locaux, au ministre de la Santé et des Services sociaux ainsi qu'au premier ministre pour dénoncer l'état scandaleux des services psychiatriques dans leur localité et pour présenter des revendications énergiques.

Ce qui importait pour ces défenseurs de la communauté, c'était que le gouvernement investisse dans des services psychiatriques de meilleure qualité et mieux adaptés à leur collectivité, et qui compléteraient le soutien fourni aux malades mentaux par les familles et par les bénévoles. L'hôpital local et ses piètres installations psychiatriques constituaient l'objet immédiat de leurs critiques acerbes, et une des prémisses de leur stratégie générale était de s'adresser «au sommet», d'exercer des pressions politiques au lieu de se perdre dans 
les formalités bureaucratiques du système régional de santé et de services sociaux. Par conséquent, les animateurs de la communauté avaient peu de relations avec les administrateurs du Conseil régional et accueillaient avec méfiance leurs demandes ou leurs offres. $\mathrm{Au}$ sein de l'hôpital local, il existait des conflits entre les groupes professionnels. Depuis le début des années 80 , les travailleurs sociaux avaient vainement tenté à plusieurs reprises d'amener l'unité psychiatrique sous-développée à élargir son mode d'intervention. L'hôpital était de moins en moins en mesure de faire face au nombre croissant de patients psychiatriques dans la localité, et au syndrome connexe de la «porte tournante». Les travailleurs sociaux chargés de planifier le retour des patients dans la communauté et de collaborer avec les familles ne demandaient qu'à mettre de nouvelles solutions en cuvre, mais ils n'obtenaient aucun appui du corps médical ou d'une administration hospitalière conservatrice. Leurs relations avec les membres militants de la communauté étaient également tendues, à cause des attaques virulentes de ces derniers contre les services hospitaliers, stratégie qui empêchait les deux groupes pro-réforme de trouver un terrain d'entente.

En 1983, le Conseil régional est intervenu dans cette situation. Ayant été autorisé par le ministère de la Santé et des Services sociaux à consacrer un pour cent des budgets des hôpitaux psychiatriques dans la région à la création de services de soutien communautaire, le Conseil a nommé un coordonnateur et a accordé un budget initial récurrent d'environ $400000 \$$ dans chacune des six sous-régions de la ville, dont la banlieue ouest. Le mandat des coordonnateurs semblait assez précis: constituer un comité aviseur local et, avec l'aide de ce dernier, mettre sur pied un réseau de ressources communautaires en santé mentale. Cette politique régionale constituait une forme de décentralisation radicale, étant donné le pouvoir de recommandation accordé à chaque coordonnateur pour la répartition des fonds. Toutefois, les leaders locaux ont accueilli avec méfiance cette initiative, n'y voyant qu'une preuve que la bureaucratie gouvernementale avait le bras long. En fait, pour contrer cette "intrusion», des groupes communautaires locaux en santé mentale se sont unis et ont créé une table de concertation.

Il existait beaucoup d'ambiguîté au sujet de l'appartenance du coordonnateur sousrégional, et du comité aviseur. Le comité regroupait en nombre égal des représentants des services publics et des groupes communautaires qui avaient été recrutés par le coordonnateur. Le premier coordonnateur avait été nommé par le Conseil régional, et, officiellement, il était un employé du centre des services sociaux du secteur (qui administrait en fiducie le nouveau budget de développement local), mais il était entendu dans la localité que la nomination de tout nouveau coordonnateur devait être officieusement approuvée par le comité aviseur existant ${ }^{9}$. Dans de telles conditions, on ne savait pas si le nouveau coordonnateur était un agent du Conseil régional introduit dans la communauté pour coordonner la création locale de ressources communautaires avec l'aide d'un comité aviseur, ou si le cornité constituait un instrument puissant offert à la communauté pour qu'elle puisse diriger le coordonnateur dans son rôle d'agent de changement et de médiateur avec le Conseil régional. Le Conseil régional et les leaders locaux ont adopté le premier point de vue, tandis que d'autres acteurs communautaires ont adhéré au second et ont constitué par la suite un «contre-leadership» en collaboration avec certains professionnels de l'hôpital.

En définitive, le comité aviseur local comme la table de concertation ont servi à mettre en lumière, au sein de la localité, des divisions et des alliances demeurées cachées jusque là. Tout d'abord, bien que les leaders communautaires les plus en vue aient refusé de participer directement au travail du comité aviseur, préférant user de leur influence de

9. Voici certaines conditions que ne partageaient pas toutes les localités de la région en question: une représentation égale des groupes communautaires et des établissements publics; la participation du comité local au choix du coordonnateur, même lorsqu'un nouveau coordinateur devait être nommé après la création du comité. Ces différences reflètent la position et les relations sociales des acteurs locaux dans le domaine de la santé mientale, ainsi que les relations entre les leaders locaux et les administrateurs du Conseil régional. Dans la banlieue ouest, il y avait un leadership communautaire fort et un leadership institutionnel faible; les chefs locaux et les administrateurs régionaux se connaissaient relativement peu, puisque la stratégie locale avait toujours été d'utiliser une médiation politique plutôt que bureaucratique. 
l'extérieur, d'autres ont jugé qu'une participation directe leur donnerait l'occasion de manipuler le comité de façon efficace. Ces stratégies différentes chez les militants locaux ont influé sur la dynamique interne de la table de concertation des groupes communautaires et l'ont empêchée de contrebalancer le comité aviseur. En second lieu, les membres du comité aviseur se sont rendus compte que leurs objectifs n'étaient pas différents de ceux de certains groupes professionnels de l'hôpital, comme les travailleurs sociaux et les membres du département de santé communautaire. Les leaders communautaires qui favorisaient une orientation professionnelle et les groupes professionnels qui favorisaient une orientation communautaire ont fait alliance, ce qui leur a permis de contrôler le comité aviseur et d'orienter ses stratégies pour la création de nouvelles ressources. À cause de l'indifférence des administrateurs de l'unité psychiatrique de l'hôpital, le comité pouvait même agir comme un catalyseur de changement au sein de l'hôpital. Il a créé des liens consultatifs avec divers groupes du personnel associés à la psychiatrie, bien que ce ne soit pas avec les psychiatres eux-mêmes, et il a recommandé le financement de projets conjoints de l'hôpital et de la communauté. Parallèlement, il a appuyé et fortement renforcé les groupes communautaires existants, même ceux qui s'opposaient à son existence.

Dans les faits, le comité est donc devenu un important agent de changement entre les mains d'une coalition locale, et il a même servi par la suite à désamorcer les conflits locaux en servant de médiateur efficace entre les groupes professionnels et communautaires. Même si le Conseil régional semblait être intervenu de façon dynamique en créant ce comité aviseur, sa stratégie subséquente pour le suivi administratif a démontré à quel point le processus de réforme échappait à son contrôle. Les comités aviseurs fonctionnaient depuis plus de cinq ans lorsque le Conseil a prétexté l'adoption de la politique officielle en santé mentale pour justifier leur remplacement par de nouvelles tables de concertation tripartites dont le rayon d'action était beaucoup plus limité. Le coordonnateur qui était chargé de créer activement des ressources communautaires a vu sa mission réduite à une vague autorisation de veiller à la coordination des ressources de la localité. De plus, le Conseil régional a centralisé le budget en santé mentale de toute la région de Montréal au lieu de le répartir entre les localités comme avant.

Cette nouvelle stratégie représentait pour le Conseil une tentative de reprendre le contrôle des processus de réforme, mais pour le sous-système local de santé mentale né dans la banlieue ouest, elle constituait un revirement ironique, mais peu important. Les rapports sociaux au sein de l'espace local s'étaient considérablement modifiés sous l'impulsion de la coalition des groupes professionnels et communautaires qui était, d'une part, surveillée par les groupes de pression traditionnels de la localité, et d'autre part, de plus en plus acceptée par l'hôpital. En outre, on avait déjà réussi à créer d'importantes ressources communautaires. Cependant, à cause de la recentralisation des contrôles, les organismes communautaires de la banlieue ouest se sont joints à d'autres organismes de la région métropolitaine pour redéfinir et justifier devant le Conseil régional et le Ministère l'autonomie locale qu'ils avaient perdue.

En résumé, l'un des aspects les plus importants des processus de réforme dans la banlieue ouest a trait aux rapports entre les groupes communautaires et professionnels, rapports extrêmement tendus, en raison de la stratégie militante utilisée par les leaders locaux plutôt que d'une différence dans les objectifs. Le comité aviseur a servi de médiateur entre ces groupes locaux et les a unis dans une coalition technocratique qui leur a servi à désamorcer les conflits politiques et idéologiques ainsi qu'à adopter et à appliquer un plan directeur au niveau local. Le comité n'a pourtant pas réussi à servir de médiateur entre le Conseil régional et la localité comme le voulait le Conseil. Lorsque ce dernier a dissous le comité et renvoyé le coordonnateur, pour en nommer un autre dont l'allégeance n'était pas ambiguë, les conflits locaux étaient suffisamment désamorcés pour que la table de concertation locale puisse jouer efficacement le rôle de défenseur de la «communauté ». Avec la recentralisation des contrôles au sein du Conseil régional, «les intérêts communautaires » s'expriment maintenant dans l'optique d'une autonomie locale pour la planification de ressources. 


\section{LA BANLIEUE SUD}

Comme la banlieue ouest, la banlieue sud est un vaste territoire situé près de Montréal, mais elle est sous l'autorité d'un conseil régional pour la gestion de la santé et des services sociaux différent du précédent. Le territoire, qui s'étend jusqu'à la frontière américaine, est parsemé à parts égales de petites villes de banlieue et de centres semiurbains. La population, qui comprend surtout des travailleurs industriels et des travailleurs des services de la classe moyenne, a connu une forte croissance depuis les années 70. Contrairement à la banlieue ouest, jusqu'à la fin des années 80 , ce territoire, pourtant très animé, est demeuré presque complètement dépourvu de services de santé, notamment de services en santé mentale.

Avant 1988, il n'y avait aucun hôpital. Un certain nombre de CLSC comblaient ce vide; un d'entre eux, notamment, avait pris sur lui d'occuper une place dans le domaine de la santé mentale en se donnant le mandat inhabituel d'assurer un service téléphonique d'urgence vingt-quatre heures par jour. Depuis sa création au début des années 70 , ce CLSC' s'était occupé activement de la vie communautaire, dans des domaines comme les coopératives de logement, par exemple. C'était la même personne qui avait créé et dirigé le CLSC pendant toutes ces années, et à l'importance de son leadership dans la communauté s'ajoutait sa position influente au sein du réseau régional de santé et de services sociaux. Selon ses propres mots, le directeur du CLSC avait une «appartenance» à la communauté plutôt qu'au réseau public des services de santé, et «son» CLSC agissait surtout à titre d'agent local.

Le premier conflit local dans le domaine de la santé mentale, comme dans les autres domaines, a opposé «l'action communautaire», sous le leadership du CLSC, au «développement technocratique », sous le leadership du Conseil régional. Un aspect important de ce conflit était le fait que les intervenants locaux s'identifiaient à une tradition «radicale», c'est-à-dire à des mouvements de gauche, notamment au mouvement anti-psychiatrique. Cette opposition entre un CLSC non conformiste et l'administration régionale n'était pas un phénomène unique, notamment dans le cas des CLSC les plus anciens, puisque, en raison même de la définition d'un CLSC, il existait des tensions structurelles entre l'appartenance à la communauté locale et l'appartenance au réseau public des services de santét ${ }^{10}$.

Le Conseil régional, comme plusieurs autres, a tenté au début des années 80 d'amorcer une réforme des services en santé mentale. Bien qu'incapable d'adopter la même stratégie que le conseil d'une région abritant des hôpitaux psychiatriques dont des fonds pouvaient systématiquement servir à créer de nouvelles ressources, il a néanmoins été un des premiers conseils à publier un plan régional pour la mise sur pied de ressources communautaires en santé mentale (1982). Il a pris cette initiative en partie parce que l'absence de ressources psychiatriques traditionnelles sur son territoire l'obligeait à trouver d'autres stratégies pour servir sa clientèle, et en partie parce qu'il avait nommé à la tête des services en santé mentale une directrice déterminée et animée d'un esprit communautaire $^{11}$. Le plan de 1982 devait néanmoins rester lettre morte tant qu'il n'y aurait pas de budget pour son application.

En l'absence de fonds suffisants pour mettre tout le plan en œuvre, la directrice de la division de la santé mentale du Conseil régional a entrepris de lancer son programme de

10. En ce sens, les CLSC constituent une illustration intéressante de l'ambiguité de la distinction État/société civile. Pour une analyse plus vaste de l'ambivalence structurelle des CLSC, voir GODBOUT et GUAY (1989) et pour ce qui concerne cette même question dans le cadre de la réforme en santé mentale en particulier, DESBIENS (1992).

11. Notons un détail significatif au sujet de cette personne: elle fut plus tard mutée au Conseil régional de Montréal et a présidé à la planification et à la mise en ceuvre de la politique de décentralisation du Conseil pour la création de ressources communautaires en santé mentale, c'est-à-dire qu'elle a veillé à la nomination des coordonnateurs locaux et des comités aviseurs. La mobilité des personnes clés est souvent un important facteur de médiation entre différents lieux de réforme et contribue à une certaine globalisation des processus, sinon des résultats. 
réforme en faisant appel à la collaboration des leaders locaux dans le domaine de la santé et des services sociaux, comme le directeur du CLSC local. Celui-ci a donc eu la possibilité de créer un mouvement local en santé mentale, c'est-à-dire un mouvement qui pourrait bénéficier des ressources allouées par le système, mais qui évoluerait en définitive sous le leadership local et qui refléterait le discours militant de ce leadership. Conformément aux traditions communautaires locales, le directeur du CLSC s'est allié avec des personnes connues qu'il jugeait compétentes dans les domaines de la santé mentale et du militantisme communautaire.

Le Conseil régional est parvenu par la suite à financer partiellement une première ressource communautaire, un projet d'hébergement collectif, mais les leaders locaux ont obtenu des fonds additionnels de sources diverses, ce qui leur a donné une certaine autonomie par rapport au Conseil. Toutefois, à cause de son approche anti-psychiatrique, la ressource a été boycottée par les hôpitaux et par les services sociaux desservant la banlieue sud, qui ont refusé d'y envoyer leurs patients ou leurs clients. Grâce à l'appui constant du CLSC, le nouveau mouvement local a quand même pu nouer des liens précieux (bien que cachés) avec certains autres établissements du réseau, et même avec le Ministère, comme l'illustre l'épisode ci-dessous.

En 1986, le Ministère a décidé de construire un hôpital dans la banlieue sud. Ce projet a suscité une foule d'activités et le CLSC y a joué un rôle clé. Pour planifier la future unité psychiatrique de l'hôpital, on a créé un comité composé d'experts professionnels et administratifs parmi lesquels figurait le directeur du CLSC local. Dans un geste de médiation stratégique, ce dernier a fait nommer au comité un militant du mouvement local en santé mentale. S'inspirant fortement des discours communautaires qui circulaient à tous les niveaux du système et qui étaient défendus avec vigueur par les militants de la banlieue sud, le comité a adopté une recommandation selon laquelle 1,2 million de dollars seraient puisés dans le budget des soins psychiatriques du nouvel hôpital et réservé à la création de services de soutien dans la communauté. Le Ministère a approuvé ce plan et des fonds ont été mis de côté pour le projet.

Ce généreux budget réservé à la création de ressources communautaires en santé mentale dans la banlieue sud faisait triompher l'autonomie locale. En contraste, le Conseil régional devait se contenter de fonds de sources diverses, ne s'élevant à pas plus de quelques centaines de milliers de dollars pour toute la région (neuf localités en total). Toutefois, comme c'était la division des services hospitaliers du Ministère qui veillait directement à la planification de l'hôpital de la banlieue sud, ni le Conseil régional ni la division de la santé mentale du Ministère n'avaient été mis au courant des résultats de la stratégie locale pour l'obtention de l'autonomie financière dans ce domaine. Lorsque le premier versement arriva en 1990, le Conseil régional a été choqué et s'est opposé vivement à ce qu'il soit tout dépensé dans cette seule localité. À ce moment, la province avait déjà adopté sa politique en santé mentale et le Conseil avait présenté son plan régional. On jugeait que le versement direct à une localité d'un nouveau budget en santé mentale (par l'entremise de son hôpital), sans tenir compte du plan régional, invalidait l'année de planification du comité tripartite. Les leaders locaux de la banlieue sud, comme le directeur du CLSC qui était aussi membre du comité tripartite, ont dû admettre la justesse de ce raisonnement. Enfin, le Conseil a permis à la banlieue sud de garder ce premier versement, à condition de recevoir les versements subséquents qui serviraient à la mise en application de son plan régional.

On trouve beaucoup d'autres exemples d'une mobilisation autonome en faveur de projets locaux sans que les militants passent par les autorités régionales. Ainsi le directeur du CLSC local était un membre important du comité tripartite que le Conseil avait formé pour établir son plan régional. Bien que membre officiellement d'un groupe représentant le réseau public, il a organisé avec succès un mouvement d'opposition pour empêcher que le système ne soit géré par les psychiatres et, avec l'appui de ses «partenaires » au sein de la communauté, il a obtenu qu'on accorde un rôle prédominant aux CLSC et aux groupes communautaires dans le plan régional. Parallèlement, les militants de la communauté 
locale sont devenu des figures clés dans les associations régionales et provinciales, comme le Regroupement des ressources alternatives en santé mentale et l'Association des groupes et des intervenants en défense de droits. De tels réseaux, qui s'étendent par-delà les frontières d'une localité, n'accroissent pas seulement l'autonomie locale, mais font également que le Conseil régional dépend des leaders locaux chaque fois qu'il veut amorcer une action au niveau local.

En somme, dans la banlieue sud, les leaders locaux ont contrôlé les processus de réforme et ont pu s'assurer que l'intervention technocratique du Conseil régional n'allait pas à l'encontre de leur radicalisme. Ils y sont parvenus en tirant parti, dans leur stratégie, de la position de médiation inhérente à leur CLSC, à la fois principal organisateur communau taire et premier représentant du réseau public dans la localité. Au lieu de lutter directement contre le Conseil régional pour diriger les processus de réforme locaux, le directeur du CLSC a noué des liens importants avec d'autres lieux globalisants comme le Ministère et les associations provinciales. Ce contrôle commun unique a permis l'utilisation, comme instrument de réforme, d'une idéologie radicale au lieu de la technocratie. Ainsi, toutes les ressources en santé mentale qui veulent desservir la communauté passent par les leaders locaux et par le crible de leur idéologie. Par exemple, lorsque le Conseil régional a distribué des fonds aux groupes d'entraide dans le cadre de la politique provinciale en santé mentale, un groupe particulier n'a pu se faire reconnaître parce qu'il ne répondait pas aux normes idéologiques locales.

\section{LA PETITE VILLE INDUSTRIELLE}

Le territoire englobant cette petite ville, qui se trouve dans la même région que la banlieue sud, offre un cas révélateur parce que son processus de réforme a été à la fois similaire et différent par rapport à celui de la banlieue sud. Quelque peu éloigné de la métropole, le territoire couvre une superficie géographique plus vaste mais moins densément peuplée que les banlieues sud et ouest, avec deux petites villes formant deux centres d'activités distincts. Bien qu'il s'agisse d'un territoire industriel doté d'une population ouvrière, il existe de grandes différences entre ces localités. Dans l'ensemble, par rapport à la banlieue sud et à la banlieue ouest, le taux de chômage est supérieur, le niveau d'éducation légèrement inférieur et la population un peu plus âgée.

La petite ville la plus importante du territoire est le siège des services de santé et des services sociaux et possède un hôpital, un centre de services sociaux et un département de santé communautaire. L'unité psychiatrique de l'hôpital n'est pas très développée, mais elle a collaboré étroitement, avec les travailleurs sociaux et les travailleurs en santé communautaire, à la mise sur pied de services de soutien dans la communauté. Dans les années 80 , on a créé des familles d'accueil psychiatriques, un hôpital de jour et un centre de réadaptation comme compléments des services hospitaliers et sociaux, en utilisant les budgets de ces services ou des budgets spécialement alloués à cette fin. Dans ce milieu, où les soins en santé mentale sont axés sur l'hôpital, il existe depuis 1984 un seul organisme communautaire offrant des services d'hébergement alternatifs et il est financé par le Conseil régional dans le cadre de son plan de 1982.

Par rapport aux banlieues sud et ouest, la caractéristique originale de cette petite ville est le fait que les leaders locaux se retrouvent presque tous au sein des établissements du réseau public de santé et de services sociaux. Pour ce groupe intime d'organisateurs de ressources, la signification professionnelle de leur participation à la mise sur pied de services en santé mentale s'exprime en ces termes: «... moi j'aime ça quand ça bouge puis que je peux faire des projets. Si je fais des projets et que ça débloque pas, $\mathrm{j}$ 'ai pas de raison de rester là; la routine à un moment donné c'est pas ça que je cherche...». Par contre, cette forme d'action comporte également un aspect institutionnel, puisque ces professionnels ne sont pas des acteurs sociaux autonomes, mais agissent sous l'autorité des administrateurs des établissements où ils travaillent. Comme membres du réseau régional de santé et de services sociaux, ils bénéficient de liens à la fois officiels et 
officieux entre eux et aussi avec le Conseil régional. Par exemple, un professionnel local a été muté dans la division de la santé mentale du Conseil régional, et a pu s'assurer ainsi qu'un projet de la petite ville qu'il parrainait soit appuyé par le centre.

En outre, comme dans la banlieue sud, il n'existe pas de véritable concurrence au niveau du leadership local dans le domaine de la santé mentale. Les professionnels institutionnels de la petite ville ne se sont pas sentis menacés par le dynamisme croissant, partout au Québec, du discours communautaire sur la santé mentale, puisque leur stratégie qui consistait à créer des programmes complémentaires hors des murs de l'hôpital pouvait elle-même se réclamer de ce discours. Lorsqu'ils mettaient sur pied des programmes, leur principal défi a toujours été d'obtenir les ressources matérielles nécessaires des directeurs de leurs établissements. Il s'agissait d'une affaire interne, d'un enjeu organisationnel. En d'autres mots, l'espace local où fonctionnent ces organisateurs était leur propre établissement, ou, tout au plus, le réseau public et le Ministère de la Santé et des Services sociaux.

Dans certains cas, les préoccupations des organisateurs n'ont pas coïncidé avec celles de leurs administrateurs institutionnels, et il s'agissait là d'un conflit classique entre professionnels et bureaucrates. En outre, lorsque le Ministère a commencé à participer de plus en plus activement au processus de réforme et s'est mis à allouer des fonds pour certains projets communautaires en santé mentale, il a aussi imposé, pour la répartition de ces fonds, de nouvelles règles qui ont perturbé les méthodes intra-réseau habituelles car elles exigeaient la participation communautaire. La mise sur pied d'une «équipe de crise» locale chargée des cas d'urgence psychiatrique illustre l'effet combiné de ces deux dynamiques. En 1987, le Ministère a annoncé son intention d'établir un seul centre de crise dans une autre localité de la région. Les professionnels de la petite ville se sont unis pour lancer une campagne visant à obtenir une partie du budget pour leur propre localité. À ce stade, ils ont été appuyés par les directeurs généraux de leurs établissements qui les ont aidés à obtenir l'appui du Ministère et le budget désiré. Le Conseil régional a été chargé par le Ministère de coordonner le processus d'implantation. Il a donc mis sur pied un comité local qui regroupait les professionnels les plus en vue dans le domaine de la santé mentale dans la petite ville, mais aussi des représentants des groupes communautaires locaux, conformément aux règles du Ministère. Entre-temps, pour des raisons purement bureaucratiques, c'est l'hôpital qui conservait le budget en fiducie.

Le comité d'implantation, dont les membres n'avaient pas l'habitude de travailler ensemble, ne parvenait pas à un consensus au sujet de l'organisme (communauté ou CLSC) qui administrerait la nouvelle équipe de crise, même si tous s'entendaient pour ne pas placer celle-ci sous le contrôle de l'hôpital. Toutefois, lorsque les directeurs généraux locaux ont eu vent de l'orientation que prenait le comité, ils ont autorisé sur-le-champ le versement du budget à l'hôpital, prétextant que le comité était dans une impasse et que la création d'une équipe de crise serait retardée indéfiniment si l'hôpital n'agissait pas. Au milieu des protestations outrées des membres du comité, l'hôpital a confié l'équipe de crise à un CLSC tout en continuant de gérer son budget, dont la plus grande partie servait à payer le salaire d'un coordonnateur rattaché à l'hôpital. Dans ce cas précis, l'espoir des professionnels de créer un nouveau type de service autonome a été annihilé par la détermination d'une institution de conserver son budget, et donc de contrôler l'ensemble des programmes. Comme le faisait remarquer un organisateur professionnel, «le pouvoir est revenu là où il est officiellement $\%$.

Depuis ce temps, les budgets alloués à la petite ville en vertu de la politique provinciale en santé mentale et du plan régional du Conseil ont continué de passer par le réseau public local, par exemple, pour créer une série de familles d'accueil d'urgence parrainées par le centre des services sociaux. Toutefois, trois événements favoriseront peut-être l'apparition d'un contrepoids à la puissante coalition du réseau : (1) la création par le Conseil de tables de concertation tripartites locales pour la mise en cuvre de la politique en santé mentale; (2) les fortes tensions entre l'hôpital et les CLSC à cause des événements entourant la création de l'équipe de crise et à cause des dispositions insatisfaisantes imposées par l'hôpital, si bien que les CLSC, dont la position est toujours ambiguë, 
sont plus portés maintenant à s'allier avec les groupes communautaires qu'avec les autres établissements du réseau; (3) l'établissement, sous l'égide des leaders de la banlieue sud, du Regroupement des ressources alternatives de la région qui accrôt le pouvoir des groupes communautaires de la petite ville grâce aux liens créés avec des groupes similaires en dehors de leur localité. Le processus de réforme ne fait peut-être que débuter dans la petite ville, puisque les acteurs locaux disposent maintenant d'un forum (les tables de concertation) et qu'un enjeu local semble enfin se cristalliser, soit la lutte entre les institutions et la communauté pour le contrôle des ressources qui seront développées en santé mentale dans l'avenir.

En résumé, dans les années 80 , la mise sur pied de nouveaux programmes dans la petite ville correspondait à l'expansion et à la diversification internes des services du réseau, conformément aux aspirations professionnelles et administratives des fournisseurs de services publics. Même s'ils s'inspiraient du discours communautaire, ces programmes n'ont pas entraîné une participation accrue de la communauté à la planification et à la création de ressources en santé mentale. En outre, les stratégies des professionnels et des administrateurs du réseau n'étaient même pas considérées comme des instruments de réforme, mais plutôt comme des moyens de faire progresser les institutions et les groupes professionnels. C'est là une illustration de «la promiscuité » (Gordon, 1980) de ce que l'on pourrait appeler «les stratégies communautaires» en santé mentale: différents acteurs se servent différemment de toute la gamme des discours communautaires au Québec dans les années 80 pour parvenir à des fins fort différentes.

\section{LA STRUCTURATION DES SOUS-SYSTÈMES LOCAUX DANS LE CONTEXTE DE L'INTERVENTION DE L'ÉTAT}

Nous avons amorce notre propos avec trois grandes hypothèses: (1) les processus de réforme ne relèvent pas strictement de l'État, mais l'État intervient plutôt dans les instruments de changement social; (2) par conséquent, les décisions et les actions du centre, ou des agents de l'État, ne suffisent pas à expliquer les résultats d'une réforme; (3) le point de départ de notre enquête doit être un espace local spécifique où se déroulent les processus de réforme, parce que ces lieux d'interaction locaux intègrent la totalité des conditions immédiates et globales qui précipitent les stratégies et les résultats réels du changement.

Qu'est-ce qu'une telle méthode nous a permis d'observer au sujet de la réforme du systèrne de la santé mentale au Québec? Premièrement, il est clair que les acteurs locaux maîtrisent jusqu'à maintenant les processus de réforme dans les trois localités étudiées. Si ceci est assez évident dans la banlieue sud et dans la petite ville, où les militants locaux et les professionnels locaux empiètent respectivement sur leur systèmes locaux, la domination du local dans la banlieue ouest est plus subtile. Car ici, le local s'est approprié les agents de l'État (le coordonnateur local et le comité aviseur établis par le Conseil régional), c'est-à-dire que ces derniers étaient entraînés dans les enjeux et dans les rapports sociaux locaux au point de ne plus être considérés comme des agents de l'État. Cette interprétation est corroborée par la réponse qu'a provoquée cette situation: le Conseil régional a adopté une nouvelle stratégie consistant à réaffirmer l'appartenance du coordonnateur au Conseil, à éliminer le comité aviseur et à faire valoir la hiérarchie centrelocal. Toutefois, le résultat ultime de cette tactique demeure incertain, puisque cette première intervention a déjà contribué à unifier les acteurs locaux et à orienter leurs stratégies vers le Conseil régional, acteur bureaucratique auparavant boudé par la localité.

Deuxièmement, nous avons découvert que les agents de l'État peuvent agir de façons très diverses dans des espaces locaux non étatiques. La forme de leur participation dépend de la forme de médiation entre les lieux divers où se déroulent les processus de réforme (par exemple entre un Conseil régional et une localité sous son autorité), chaque lieu possédant son propre ensemble d'acteurs, de relations et de conditions d'action immédiates et proches. Dans la banlieue ouest les médiateurs ont été les acteurs (coordinateur) ainsi que les structures (comité aviseur) à la fois choisis par la localité et insérés 
dans celle-ci avec un mandat précis, mais tel n'a pas été le cas dans la banlieue sud et dans la petite ville. Le CLSC et son directeur ont été les principaux instruments de médiation dans la banlieue sud. Imbriqué à la fois dans la communauté et dans le réseau public, le CLSC ne constituait pas un instrument potentiel pour l'État et pour la localité (comme l'a fait le coordonnateur et le comité aviseur de la banlieue ouest), mais il permettait plutôt à son directeur d'avoir intimement accès aux deux lieux. En conséquence, les acteurs locaux et les acteurs du Conseil régional dépendaient des relations et de l'influence $\mathrm{du}$ directeur. Une comparaison des deux formes de médiation que nous venons de décrire nous permet d'avancer l'hypothèse suivante: le modèle instrumental de médiation (banlieue ouest) dépend davantage de l'État qui, en bout de ligne, contrôle la structure opportune, tandis que le modèle de la médiation imbriquée (banlieue sud) dépend davantage du bon vouloir et des stratégies de la personne qui occupe le lieu de médiation.

Troisièmement, dans les cas où les agents de l'État ne se mêlent pas des enjeux et des rapports locaux, on peut se demander s'il se produit vraiment une réforme. Nous avons défini précédemment la réforme comme toute réorganisation sociale, politique ou économique faisant appel à l'intervention de l'État; il faut peut-être clarifier maintenant cette définition en soulignant que nous faisons référence à une réorganisation des rapports sociaux, politiques ou économiques. Cela laisse sous-entendre que les processus de réforme supposent nécessairement l'arrivée de nouveaux acteurs sur la scène locale, des alliances ou des confrontations nouvelles, de nouveaux rapports d'autonomie ou de dépendance ou d'autres développements similaires. Ce genre de réorganisation peut-il résulter d'une simple injection de fonds dans une localité, ou encore de l'imposition discursive de règlements (par exemple, «l'adoption» d'une politique ou d'une loi)? Vus sous cet angle, les événements qui se sont déroulés à la petite ville sont les plus révélateurs.

L'arrivée de fonds pour de nouveaux projets n'a pas contribué en soi au processus de réforme dans la petite ville, mais a simplement permis aux acteurs locaux de renforcer le statu quo. Même lorsqu'il existait des règlements relatifs au type de ressources pour lesquelles les fonds étaient accordés, ces règlements dépendaient du classement des ressources en ressources «communautaires » ou non, et ce classement dépendait à son tour du discours. Toutefois, non seulement existait-il divers discours communautaires pouvant servir à classer les ressources en santé mentale, mais il y avait aussi de nombreux moyens administratifs de modifier les descriptions des ressources pour qu'elles soient conformes aux discours officiellement sanctionnés ${ }^{12}$. Ainsi, même après que la politique provinciale en santé mentale eût réglementé le classement des ressources et la répartition des fonds, la marge de manouvre était assez large pour permettre des manipulations au niveau local. Dans la petite ville, les établissements publics ont continué de gérer les budgets et les programmes.

Parallèlement, ce que nous avons appelé les «structures opportunes» au niveau politique et administratif ne peuvent suffire à expliquer les processus de réforme, ou même à les orienter dans une direction donnée. Nous avons vu que, dans la région de la banlieue sud et de la petite ville, par exemple, le peu de fonds publics disponibles pour la création de ressources communautaires au moment où les établissements du réseau public subissaient des réductions budgétaires, a eu deux résultats différents selon les conditions locales: l'intervention de nouveaux acteurs (contestataires) ou le renforcement du statu quo. Pour ce qui est de la banlieue ouest, ce que certains acteurs locaux ont considéré comme une structure opportune (soit le coordonnateur local, le comité aviseur et le budget autorisés par le Conseil) pour la réalisation autonome de changements systématiques, est rapidement disparu lorsque les administrateurs du Conseil régional ont découvert que cette structure fonctionnait sans se préoccuper de leurs définitions de la situation et de leurs objectifs.

12. Pour une analyse des moyens utilisés pour rendre différents types de ressources conformes au discours officiel dans le contexte de la réforme de la santé mentale au Québec, voir WHITE et MERCIER (1991a et b). 
Enfin, nous devons considérer la signification du local pour comprendre des processus de réforme plus globaux. Il existe deux possibilités. D'une part, nous pouvons apprécier le local comme un site de réforme sociale historiquement significatif, comme le laisse entendre Klein (1989, p. 189):

ILes nouveaux espaces de développement qui se configurent au niveau local, soient-ils découpés par l'Etat, par les pouvoirs locaux ou par les mouvements sociaux, constituent les nouveaux lieux de «convergence», ce qui ne veut pas dire de consensus, d'acteurs sociaux d'ancrage différent, tout comme le fut jadis l'État nation.

Vue sous cet angle, l'importance du local comme premier lieu de réforme est un phénomène de l'État postprovidence qui cherche à se désengager de la gestion sociale, et dans la mesure où il donne naissance à des effets fragmentés et distincts, il est peut-être même un phénomène sociopolitique postmoderne.

D'autre part, le local pourrait être considéré uniquement comme un instrument d'analyse. Dans ce cas, nous serions éventuellement amenés à nous concentrer sur les effets cumulatifs des processus de réforme locaux, que ces effets soient intentionnels ou non. Nous partons ainsi de l'hypothèse selon laquelle les événements locaux ne sont que des moments dans un processus plus long et plus global qui acquiert de la cohésion à partir des discours et des pratiques qui traversent certains espaces locaux et qui agissent comme agents de médiation. Si cela est vrai, nous pouvons nous attendre, comme cela s'est produit pour la médicalisation dans une génération de réforme antérieure, à des processus permanents qui finiront par unifier le domaine de la santé mentale sous un nouveau déguisement «communautaire».

\author{
Deena WHITE \\ Département de sociologie \\ et Groupe de recherche sur les \\ aspects sociaux de la prévention \\ Université de Montréal \\ C.P. 6128, Succ. «A» \\ Montréal (Québec) \\ Canada $\mathrm{H} 3 \mathrm{C}$ 3J7
}

\title{
RÉSUME
}

L'article se veut un apport a la constitution d'une sociologie des processus de reforme. Nous ne prenons pas comme point de départ les programmes ou les acteurs de l'État mais, afin de rendre compte des effets diversifiés, pour ne pas dire arbitraires, des politiques sociales, nous nous intéressons plutôt à la façon dont les acteurs sociaux locaux s'approprient des processus de réforme. Nous présentons les résultats d'une étude de cas multiples axée sur les développements qui se sont produits dans le réseau des soins de santé mentale de trois localités du Québec, dans le contexte d'une réforme provinciale du système de santé mentale. Nous concluons en présentant un certain nombre d'hypothèses relatives à la structuration des sous-systèmes locaux face à l'intervention étatique.

\section{SUMMARY}

This article aims to contribute to the constitution of a specifically sociological enquiry into reform processes. Rather than using state programs or actors as starting points for an investigation of policy development or implementation, we focus on the appropriation of policies and reform processes by local social actors, and the shaping of outcomes by local social relations and other local conditions. The fragmented and incoherent nature of reforms are explained through the example of a major mental health care reform in Quebec, showing its dissimilar progress and impact in three different localities. We conclude with a number of hypotheses regarding the structuration of local sub-systems in contexts of state intervention.

\section{RESUMEN}

Este articulo pretende ser un aporte a la constitución de una sociologia de los procesos de reforma. Nosotros no tomamos como punto de partida los programas o los actores del Estado sino que, a fin de dar cuenta de los 
efectos diversificados, por no decir arbitrarios, de las políticas sociales, nosotros nos interesamos más bien a la manera por la cual los actores sociales locales se apropian de los procesos de reforma. Presentamos los resultados de un estudio de casos múltiples orientado sobre los desarrollos producidos dentro de la red sanitaria en salud mental de tres localidades de Quebec, dentro del contexto de una reforma provicial del sistema de salud mental. Nosotros concluímos presentando un cierto número de hipótesis relativas a la estructuración de los sub-sistemas locales frente a la intervención estatal.

\section{BIBLIOGRAPHIE}

ALFORD, Robert (1975), Health Care Politics, Chicago, University of Chicago Press.

ALINSKY, Saul (1969), Reveille for Radicals, New York, Vintage Press.

ALINS KY, Saul (1972), Rules for Radicals, New York, Random House.

BOUDREAU, Françoise (1987), "The making of mental health policy, the 1980's and the challenge of sanity in Québec and Ontario», Canadian Journal of Community Mental Health, vol. 6, $\mathrm{n}^{\circ}$ 1, pp. 27-46.

BOURDIEU, Pierre (1980), Question de sociologie, Paris, Éditions du Seuil.

COHEN, A.P.(1985), The Symbolic Construction of Community, Toronto, Tavistock Publishers.

COOPER, David G. (1967), Psychiatry and Anti-Psychiatry, Toronto, Tavistock Publishers.

DESBIENS, Francine (1992), Le rôle des CLSC dans le processus d'établissement d'un plan d'organisation des services en santé mentale, mémoire de maîtrise, Département de Communications, Université du Québec à Montréal.

EVANS, Peter B., Dietrich RUESCHEMEYER et Theda SKOCPOL (1985), Bringing the State Back In, Cambridge, Cambridge University Press.

FOUCAULT, Michel (1961), Histoire de la folie a l'âge classique, Paris, Plon.

FOUCAULT, Michel (1963), Naissance de la clinique. Une archéologie du regard médical, Paris, Plon.

FOUCAULT, Michel (1980), Power/Knowledge, Brighton, Harvester Press.

GIDDENS, Anthony (1981), A Contemporary Critique of Historical Materialism, Los Angeles, University of California Press.

GIDDENS, Anthony (1984), The Constitution of Society, Los Angeles, University of California Press.

GIDDENS, Anthony (1987), The Nation-State and Violence, Los Angeles, University of California Press.

GODBOUT, Jacques et Jérome GUAY (1989), Le communautaire public: Le cas d'un CLSC, Montréal, INRS-Urbanisation, Études et documents 62.

GODBOUT, Jacques (1983), La participation contre la démocratie, Montréal, Éditions Saint-Martin.

GOFFMAN, Erving (1961), Asylums: Essays on the Social Situation of Mental Patients and Other Inmates, Harmondsworth, Penguin.

GORDON, Colin (1980), «Afterword» in Michel Foucault (1980), Power/Knowledge, Brighton, Harvester Press, pp. 229-259.

GOUGH, Ian (1979), The Political Economy of the Welfare State, Londres, Macmillan Press.

HAYEK, F.A. (1973), Economics, Freedom and Representative Government, Londres, Institute of Economic Affairs.

HELD, David (1989), Political Theory and the Modern State, Stanford, California, Stanford University Press.

HINDESS, Barry (1982), «Power, Interests and the Outcomes of Struggles », Sociology, vol.16, $\mathbf{n}^{\circ} 4$, pp.198511.

HINDESS, Barry (1986), «Actors and Social Relations » in Mark L. Wardell et Stephen P. Turner (sous la direction de), Sociological Theory in Transition, Boston, Allen and Unwin, pp. 113-126.

HINDESS, Barry (1988), Choice, Rationality, and Social Theory, Londres, Unwin Hyman.

JALBERT, Lizette (1991), «La décentralisation: enjeux et perspectives ", in Louis Maheu et Arnaud Sales (sous la direction de), La recomposition du politique, Montréal, P.U.M., pp. 251-272.

JALBERT, Lizette (1992), "L'État ancré ou les frontières de la démocratie », in Gérard Boismenu, Pierre Hamel et Georges Labica (sous la direction de), Les formes modernes de la démocratie, Montréal, P.U.M., pp. 83-112.

KLEIN, J-L. (1989), «Développement régional et espace local: vers une régulation territorialisée », Revue internationale d'action communautaire, vol. 22/62, pp. 189-196.

LOWI, Theodore (1979), The End of Liberalism, New York, W.W. Norton.

MARKS, I. et A. SCOTT, (éd.) (1990), Mental Health Care Delivery: Innovations, Impediments and Implementation, Cambridge, Cambridge University Press.

MARRIS, Peter (1987), Meaning and Action. Community Planning and Conceptions of Change, Londres, Routledge and Kegan Paul.

MÉDARD, Jean-François (1961), Communauté locale et organisation communautaire aux États-Unis, Paris, Armand-Colin.

MELUCCI, Alberto (1989), Nomads of the Present, Philadelphia, Temple University Press.

MINISTÈRE DE LA SANTÉ ET DES SERVICES SOCIAUX (1989), Politique de santé mentale, Québec, Gouvernement du Québec.

MOWBRAY, Martin (1985), «The Medicinal Properties of Localism: A Historical Perspecitve», in R. Thorpe, et J. Petruchenia (sous la direction de), Community Work or Social Change?, Londres, Routledge and Kegan Paul, pp. 54-69. 
OFFE, Claus (1984), Contradictions of the Welfare State, Londres, Hutchison. OFFE, Claus (1985), Disorganized Capitalism, Cambridge, Polity Press.

RUESC.HEMEYER, Dietrich et Peter B. EVANS (1985) \& The Sate and Economic Transformation: Toward an Analysis of the Conditions Underlying Effective Intervention", in Peter B. Evans, Dietrich Rueschemeyer et Theda Skocpol (sous la direction de), Bringing the State Back In, Cambridge, Cambridge University Press, pp. 44-77.

SALES, Arnaud (1991), «Privé, public et société civile : champs sociaux et structures du pouvoir », in Louis Maheu et Arnaud Sales (sous la direction de), La recomposition du politique, Montréal, P.U.M. pp.4368.

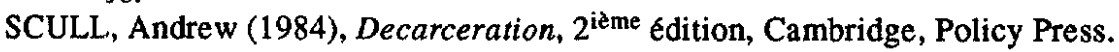

SKOCPOL, Theda (1985), «Bringing the State Back In: Strategies of Analysis in Current Research», in Peter B. Evans, Dietrich Rueschemeyer et Theda Skocpol (sous la direction de), Bringing the State Back In, Cambridge, Cambridge University Press, pp.3-37.

SZASZ, Thomas (1977), Le Mythe de la maladie mentale, Paris, Payot.

THORPE, Rosamund (1985), "Community work and ideology: an Australian perspective», in R. Thorpe et J. Petruchenia (sous la direction de), Community Work or Social Change?, Londres, Routledge and Kegan Paul, pp. 3-23.

TOMLINSON, Dylan (1991), Utopia, Community Care and the Retreat from the Asylums, Phildelphia, Open University Press.

TOURAINE, Alain (1973), Production de la société, Paris, Éditions du Seuil.

URRY, John (1982), «Some Themes in the Analysis of the Anatomy of Contemporary Capitalist Societies », Acta Sociologica, vol. 25, $\mathrm{n}^{\circ} 4$, pp. 405-418.

VALAKIÉ, P.(1988), "La décentralisation du social ou le social "négocié"», Revue internationale d'action communautaire, vol. 20/60, pp. 131-137.

WEBER, Max (1978), Economy and Society, Berkeley, Californie, University of California Press.

WHITE:, Deena (1988), Social Policy and Social Change: The Case of Occupational Health in Québec, thèse de doctorat, Département de sociologie, Université de Montréal.

WHITE, Deena (à paraître) «The Community-based Mental Health System : What Does it Mean ?», Canadian Review of Social Policy.

WHITE:, Deena et Céline MERCIER (1989), «Ressources alternatives et structures intermédiaires dans le contexte québécois », Santé Mentale au Québec, vol. 14, nº 1, pp. 69-80.

WHITE:, Deena et Céline MERCIER (1991a), «Reorienting Mental Health Systems : The Dynamics of Policy and Planning », International Journal of Mental Health, vol. 19, $\mathrm{n}^{\circ} 4$, pp. 3-24.

WHITE:, Deena et Céline MERCIER (1991b), «Coordinating Community and Public-Institutional Mental Health Services: Some Unintended Consequences», Social Science and Medicine, vol. 33, $n^{\circ} 6$, pp. 729-739.

WHITE:, Deena, Céline MERCIER et Francine DESBIENS (1991), La mise en auvre d'une politique en santé imentale: le développement des ressources communatuaires. Protocole de recherche, manuscrit inédit, Groupe de recherche sur les aspects sociaux de la prévention, Université de Montréal. 